\title{
Towers of Babel
}

\author{
Exoplanetary science warns us against the use of improper terminology, which increases the risk of \\ new discoveries being misinterpreted by researchers as well as the general public. Both the scientific \\ community and journal editors can help to avoid this significant danger.
}

Our community is a fitting example of how global collaboration leads to the advancement of science. This was the main message underlying the first issue of Nature Astronomy, as highlighted in the Editorial (article no. 0020), the Comment by Abraham Loeb and Nia Imara (article no. 0006) and the Review on planetary exploration by Andrew Ingersoll (article no. 0010). However, bringing groups of people coming from different backgrounds together is easier said than done. Even putting aside the human factor, practical problems invariably appear. The struggle to find a common ground for communication is one of the most pressing, basic and delicate among them.

\section{Some words are not neutral, but rather come with baggage that leads to misinterpretation.}

Astronomy, which is a highly interdisciplinary and globalized discipline, is particularly prone to this issue, as the following examples show. Astrochemistry brings together physicists and chemists, who use vastly different language conventions. The dialogue between the various disciplines within planetary science (astronomy, geology, climatology and so on) is sometimes complicated. More generally, many areas of astronomy involve experimental, observational and theoretical researchers, with all of the related communication problems that this cooperation implies. Even relatively restricted fields may build their own little Tower of Babel, as a blog post by Sarah Hörst on the nomenclature of planetary aerosols shows (http://go.nature.com/2iADBvg).

Within astronomy, exoplanetary science constitutes a sort of 'perfect storm' for miscommunication, for various reasons. It is a relatively new field and, as such, is still developing its own terminology. It is strongly interdisciplinary, involving stellar physicists, planetary scientists, the stellar/planetary formation and interstellar/ interplanetary medium communities, and potentially even biologists, through habitability studies. And finally, we discovered that exoplanets, far from being clones of our Solar System's planets, come in a wide range of sizes, masses, atmospheres and other characteristics. Extrasolar planetary systems are extremely diverse, so we cannot apply the SolarSystem-centric nomenclature we have developed over the last few hundred years lock, stock and barrel.

The risks related to the development of misleading jargon in exoplanetary studies is the topic of the two Comments, by William Moore and colleagues (article no. 0043) and by Elizabeth Tasker and colleagues (article no. 0042), that are included in this issue. It is interesting (and quite reassuring) that these manuscripts were submitted independently and almost at the same time, and that they have a substantial number of people behind them. Solving the communication issue is clearly perceived as a priority by the community.

As Moore et al. point out, some words are not neutral, but rather come with baggage that leads to misinterpretation. Expressions like super-Earth (or superVenus), mini-Neptune or hot Jupiter possess a specific and often misleading flavour, at least because they imply that we have a much more extensive knowledge of these bodies than we actually do. This is especially dangerous when we deal with habitability. Habitability is a slippery concept whose boundaries are continuously being revised even within our own Earth, as the constant flow of discoveries of extremophiles demonstrates. This uncertainty is magnified for rocky exoplanets, whose environments are still largely beyond our observing capabilities. The desire to find a second Earth can lead to rushed, inaccurate or downright deceitful interpretations of research. As the comment by Tasker et al. underlines, we have to be adamantly clear on what we know, what we can know, and what we don't know, especially if we want to quantify the vague notion of habitability through the use of metrics, even if it means adopting a more conservative way of expressing results.

Both Comments propose a series of solutions to overcome the status quo. Also, Nature Astronomy and other scientific journals must play an active role to counteract the use of misleading vocabulary. In the case of exoplanets, for example, Nature Astronomy will work with authors to eliminate ambiguous or faulty terminology, or a loose application of habitability metrics. This is going to be an ongoing process and thus we welcome other contributions and we encourage an active dialogue within the community through our pages.

\section{We have to be adamantly clear on what we know, what we can know, and what we don't know.}

Finally, there is an additional Tower of Babel that must be considered: communication with the outside world. In this period of relative distrust of the general public towards science, astronomy still enjoys a degree of attention and respect, as the Rosetta and New Horizons missions, for example, have demonstrated. Nevertheless, it is very easy to convey a misleading message concerning newly published research, maybe motivated by showing that public money is well spent, with the media always looking for catchy headlines. Both Comments warn against the danger of inaccurate communication between the scientific world and the public. There is a risk of saturation of flashy announcements (yet another most similar planet to Earth, yet another discovery of water on Mars ...) that could engender a sense of fatigue in the public and, in the long term, damage the credibility of our community. We cannot stop the media writing exaggerated headlines, but we can be precise and unambiguous in the terminology and in the meaning of the metrics we use as researchers and publishers. In the end, the primary responsibility to be honest falls on us. 